\title{
Global Asymptotic Stability of Impulsive CNNs with Proportional Delays and Partially Lipschitz Activation Functions
}

\author{
Xueli Song ${ }^{1}$ and Jigen Peng ${ }^{2}$ \\ ${ }^{1}$ Department of Mathematics and Information Science, Chang'an University, Xian 710064, China \\ ${ }^{2}$ School of Mathematics and Statistics, Xian Jiaotong University, Xian 710049, China \\ Correspondence should be addressed to Xueli Song; xlsung@chd.edu.cn
}

Received 15 April 2014; Accepted 26 June 2014; Published 23 July 2014

Academic Editor: Ademir F. Pazoto

Copyright (c) 2014 X. Song and J. Peng. This is an open access article distributed under the Creative Commons Attribution License, which permits unrestricted use, distribution, and reproduction in any medium, provided the original work is properly cited.

\begin{abstract}
This paper researches global asymptotic stability of impulsive cellular neural networks with proportional delays and partially Lipschitz activation functions. Firstly, by means of the transformation $v_{i}(t)=u_{i}\left(e^{t}\right)$, the impulsive cellular neural networks with proportional delays are transformed into impulsive cellular neural networks with the variable coefficients and constant delays. Secondly, we provide novel criteria for the uniqueness and exponential stability of the equilibrium point of the latter by relative nonlinear measure and prove that the exponential stability of equilibrium point of the latter implies the asymptotic stability of one of the former. We furthermore obtain a sufficient condition to the uniqueness and global asymptotic stability of the equilibrium point of the former. Our method does not require conventional assumptions on global Lipschitz continuity, boundedness, and monotonicity of activation functions. Our results are generalizations and improvements of some existing ones. Finally, an example and its simulations are provided to illustrate the correctness of our analysis.
\end{abstract}

\section{Introduction}

Cellular neural networks (CNNs) introduced by Chua and Yang $[1,2]$ have found many important applications in biology, the solving of optimization problem, image processing, and pattern recognition [3]. In fact, CNNs can be characterized by an array of identical nonlinear dynamical systems (called cells) locally interconnected in the paper [4] which presented a set of sufficient conditions ensuring the existence of at least one stable equilibrium point in terms of the template elements. As we know, time delays are inevitable in electronic implementation of CNNs [5]. However, time delays may destroy stability of the networks and even lead to the oscillation behaviors. Hence, it is necessary to study the stability of CNNs with different types of delays. Time delays may be proportional delays; that is to say, the delay function $\tau(t)=(1-q) t$ is a monotonically increasing function with respect to $t>0$, where $q$ is a constant and satisfies $0<q<1$. The type of proportional delays is usually required in Web quality of service routing decision and one may be convenient to control the network's running time according to the network allowed delays. Moreover, one can refer to the paper [6] about more information on the proportional delay engineering. Proportional delays [7-10] are unbounded time-varying ones different from constant delays [11], bounded time-varying delays [12-18], and unbounded distributed delay [19-23]. It is relatively difficult to deal with this class of the unbounded time-varying delays because none of any other assumptions are imposed on it compared with other unbounded time-varying delays, such as, unbounded distributed delays often require that the delay kernel functions $k_{i j}: \mathbb{R}^{+} \rightarrow \mathbb{R}^{+}$satisfy $\int_{0}^{\infty} k_{i j}(s) d s=1, \int_{0}^{\infty} s k_{i j}(s) d s$ $<\infty$, or there exists a positive number $\mu$ such that $\int_{0}^{\infty} k_{i j}(s) e^{\mu s} d s<\infty[20-23]$. Several stability criteria of CNNs with proportional delays have been obtained [7]. Moreover, the abrupt changes in the voltages produced by faulty circuit elements are exemplary of impulse phenomena which can affect the transient behavior of the network [24]. Hence, it is significant to discuss the stability of the CNNs with impulses and proportional delays. However, to the best 
of the authors' knowledge, few authors have handled the stability of CNNs with impulses and proportional delays.

Among the existing research results about neural networks, some activation functions are assumed to be globally Lipschitz continuous [25-30], bounded and monotonic [31], and bounded [24, 32]. However, these assumptions make these existing results unapplicable to some important engineering problems. For example, when the neural networks are used to solve optimization problems with the presence of constraints (linear, quadratic, or more general programming problems), unbounded (or nonmonotonic, non-globally Lipspchitz continuous) activations modeled by diode-like exponential-type functions are needed such that constraints are satisfied [33]. Motivated by this, we attempt to abandon these assumptions and only require activation functions to be partially Lipschitz continuous. Moreover, the relative nonlinear measure is more efficient than the nonlinear measure for exponential stability analysis of different classes of neural networks without delays where the equilibrium points are given $[20,34]$.

According to the foregoing analysis, this paper is devoted to analyzing stability of impulsive CNNs with proportional delays and Lipschitz continuous activation functions by relative nonlinear measure. The remainder of this paper is arranged as follows. Section 2 describes the model of proportion-delayed impulsive CNNs with partial Lipschitz continuous activation functions and provides its equivalent form by some transformation. Being preliminaries, Section 3 is devoted to uniqueness and exponential stability of equilibrium point of a nonlinear impulsive functional differential equation with variable coefficients and constant delays by means of relative nonlinear measure. In Section 4 , a sufficient condition is obtained for global asymptotic stability of equilibrium point of impulsive proportion-delayed CNNs with partially Lipschitz continuous activation functions by results derived in Section 3. Furthermore, an example and its simulations are presented to illustrate that our method is valid and that our derived results are new and correct. Conclusions are given in Section 5.

\section{Model Description and Its Equivalent Form}

We consider the following CNNs with impulses and multiproportional delays:

$$
\begin{gathered}
\dot{u}_{i}(t)=-d_{i} u_{i}(t) \\
+\sum_{j=1}^{n}\left[a_{i j} f_{j}\left(u_{j}(t)\right)+b_{i j} g_{j}\left(u_{j}\left(p_{j} t\right)\right)\right. \\
\left.+c_{i j} h_{j}\left(u_{j}\left(q_{j} t\right)\right)\right]+I_{i}, \quad t \geq 1, t \neq t_{k}, \\
\Delta u_{i}\left(t_{k}\right)=\mathscr{I}_{i, k}\left(u_{i}\left(t_{k}\right)\right), \quad k \in \mathbb{N}, \\
u_{i}(s)=\phi_{i}(s), \quad r \leq s \leq 1,
\end{gathered}
$$

for $i=1,2, \ldots, n$, where $n \geq 2$ is the number of cells in the networks; $u_{i}(t)$ denotes the potential of the $i$ th cell at time $t ; d_{i}>0$ represents the rate with which the $i$ th cell resets its potential to the resting state when isolated from other cells and inputs at time $t ; a_{i j}, b_{i j}$, and $c_{i j}$ denote the strengths of connectivity between the $j$ th and the $i$ th cells at time $t$, $p_{j} t$, and $q_{j} t$, respectively; $p_{j}$ and $q_{j}$ are proportional delay factors and satisfy $0<p_{j}, q_{j}<1, r=\min _{1 \leq j \leq n}\left\{p_{j}, q_{j}\right\}$ and $p_{j} t=t-\left(1-p_{j}\right) t, q_{j} t=t-\left(1-q_{j}\right) t$, in which $\left(1-p_{j}\right) t,\left(1-q_{j}\right) t$ correspond to the time delays required in processing and transmitting a signal from the $j$ th cell to the $i$ th cell, and $\left(1-p_{j}\right) t \rightarrow+\infty,\left(1-q_{j}\right) t \rightarrow+\infty$ as $t \rightarrow+\infty$; $\Delta u_{i}\left(t_{k}\right)=u_{i}\left(t_{k}^{+}\right)-u_{i}\left(t_{k}^{-}\right)$is the impulse at moments $t_{k}$ and $1=t_{1}<t_{2}<\cdots$ is a strictly increasing sequences such that $\lim _{k \rightarrow+\infty} t_{k}=+\infty ; f_{j}, g_{j}$, and $h_{j}$ are the nonlinear activation functions; $I_{i}>0$ denotes the $i$ th component of an external input source introduced from outside the network to the $i$ th cell at time $t$.

To discuss stability of the networks (1), we only assume the following.

(H) Activation functions $f_{j}, g_{j}$, and $h_{j}$ are partially Lipschitz continuous on $\mathbb{R}$ for $j=1,2, \ldots, n$.

In what follows, we plan to transform model (1) into a model what we can directly deal with. Motivated by this paper [7], we define the transformation by

$$
v_{i}(t)=u_{i}\left(e^{t}\right), \quad i=1,2, \ldots, n .
$$

(I) When $e^{t} \geq 1$ and $e^{t} \neq t_{k}$, then $t \geq 0, t \neq \ln t_{k}$ and $\dot{v}_{i}(t)=\dot{u}_{i}\left(e^{t}\right) e^{t}$; that is,

$$
\dot{u}\left(e^{t}\right)=\dot{v}(t) e^{-t} .
$$

Taking $h=e^{t}$ and then $h \geq 1$, then the transformation (2) is written as

$$
\dot{u}_{i}(h)=h^{-1} \dot{v}_{i}(t) .
$$

From (1) and (4), we derive

$$
\begin{gathered}
\dot{v}_{i}(t) h^{-1}=-d_{i} u_{i}(h) \\
+\sum_{j=1}^{n}\left[a_{i j} f_{j}\left(u_{j}(h)\right)+b_{i j} g_{j}\left(u_{j}\left(p_{j} h\right)\right)\right. \\
\left.+c_{i j} h_{j}\left(u_{j}\left(q_{j} h\right)\right)\right]+I_{i} ;
\end{gathered}
$$

that is,

$$
\begin{gathered}
\dot{u}_{i}\left(e^{t}\right)=-d_{i} u_{i}\left(e^{t}\right) \\
+\sum_{j=1}^{n}\left[a_{i j} f_{j}\left(u_{j}\left(e^{t}\right)\right)+b_{i j} g_{j}\left(u_{j}\left(p_{j} e^{t}\right)\right)\right. \\
\left.+c_{i j} h_{j}\left(u_{j}\left(q_{j} e^{t}\right)\right)\right]+I_{i} .
\end{gathered}
$$

From transformation (2), we obtain

$$
\begin{aligned}
& u_{j}\left(p_{j} e^{t}\right)=u_{j}\left(e^{t+\ln p_{j}}\right)=v_{j}\left(t+\ln p_{j}\right)=v_{j}\left(t-\tau_{j}\right), \\
& u_{j}\left(q_{j} e^{t}\right)=u_{j}\left(e^{t+\ln q_{j}}\right)=v_{j}\left(t+\ln q_{j}\right)=v_{j}\left(t-\varsigma_{j}\right),
\end{aligned}
$$

where $\tau_{j}=-\ln p_{j}, \varsigma_{j}=-\ln q_{j}$. 
By (2), (6), and (7), we enjoy

$$
\begin{aligned}
& \dot{v}_{i}(t)=e^{t}\left\{-d_{i} v_{i}(t)\right. \\
& +\sum_{j=1}^{n}\left[a_{i j} f_{j}\left(v_{j}(t)\right)+b_{i j} g_{j}\left(v_{j}\left(t-\tau_{j}\right)\right)\right. \\
& \left.\left.+c_{i j} h_{j}\left(v_{j}\left(t-\varsigma_{j}\right)\right)\right]+I_{i}\right\} .
\end{aligned}
$$

(II) When $e^{t} \geq 1$ and $e^{t}=t_{k}$, then $t \geq 0$ and $t=\ln t_{k}$. By transformation (2), we have

$$
\begin{aligned}
\Delta v_{i}(t) & =v_{i}\left(t^{+}\right)-v_{i}(t)=u_{i}\left(e^{t^{+}}\right)-u_{i}\left(e^{t}\right) \\
& =u_{i}\left(t_{k}^{+}\right)-u_{i}\left(t_{k}\right)=\mathscr{I}_{i, k}\left(u_{i}\left(t_{k}\right)\right) \\
& =\mathscr{I}_{i, k}\left(v_{i}(t)\right) .
\end{aligned}
$$

(III) When $e^{t} \in[r, 1]$, from (1) we have

$$
u_{i}\left(e^{t}\right)=\phi_{i}\left(e^{t}\right), \quad t \in[-\tau, 0],
$$

where $\tau=\max _{1 \leq j \leq n}\left\{\tau_{j}, \varsigma_{j}\right\}$. Hence, the initial functions associated with (8) are given by

$$
v_{i}(s)=\psi_{i}(s)=\phi_{i}\left(e^{s}\right), \quad-\tau \leq s \leq 0, i=1,2, \ldots, n .
$$

Conversely, let $\tau_{j}=-\ln p_{j}, \varsigma_{j}=-\ln q_{j}$ in (8); by transformation (2), then (8) can be written as (1) for $t \geq 1$ and $t \neq t_{k}, \Delta\left(u_{i}(t)\right)=u_{i}\left(t^{+}\right)-u_{i}(t)=v_{i}\left(\ln t^{+}\right)-v_{i}(\ln t)$ for $t \geq 1$ and $t=t_{k}$, and for $t \in[r, 1]$, from (10) and (11), the initial function associated with (1) is given by $u_{i}(s)=\phi_{i}(s), s \in[r, 1]$.

In conclusion, in the sense of solutions, the CNNs with impulses and multiproportional delays (1) is equivalent to the following CNNs with constant delays and variable coefficients

$$
\begin{aligned}
& \dot{v}_{i}(t)=e^{t}\left\{-d_{i} v_{i}(t)\right. \\
& +\sum_{j=1}^{n}\left[a_{i j} f_{j}\left(v_{j}(t)\right)+b_{i j} g_{j}\left(v_{j}\left(t-\tau_{j}\right)\right)\right. \\
& \left.\left.+c_{i j} h_{j}\left(v_{j}\left(t-\varsigma_{j}\right)\right)\right]+I_{i}\right\}, \quad t \neq \ln t_{k}, \\
& \Delta v_{i}(t)=\mathscr{I}_{i, k}\left(v_{i}(t)\right), \quad t=\ln t_{k}, k \in \mathbb{N}, \\
& v_{i}(s)=\psi_{i}(s), \quad s \in[-\tau, 0],
\end{aligned}
$$

for $t \geq 0$, where $\tau=\max _{1 \leq j \leq n}\left\{\tau_{j}, \varsigma_{j}\right\}, \tau_{j}=-\ln p_{j}>0$, $\varsigma_{j}=-\ln q_{j}>0, \psi_{i} \in C([-\tau, 0], \mathbb{R})$ denoting the space of all continuous functions from $[-\tau, 0]$ to $\mathbb{R}$ for $i=1,2, \ldots, n$ and $\psi=\left(\psi_{1}, \psi_{2}, \ldots, \psi_{n}\right)^{T}$.

\section{Preliminaries}

Let $n$-dimensional real vector space $\mathbb{R}^{n}$ be endowed with 1 norm $\|\cdot\|_{1}$ defined by

$$
\|x\|_{1}=\sum_{i=1}^{n}\left|x_{i}\right| \quad \text { for every } x=\left(x_{1}, x_{2}, \ldots, x_{n}\right)^{T} \in \mathbb{R}^{n}
$$

where the superscript $T$ denotes the transpose. Let $\langle\cdot, \cdot\rangle$ denote the inner product in $\mathbb{R}^{n}$ and $\operatorname{sign}(x)=\left(\operatorname{sign}\left(x_{1}\right)\right.$, $\left.\operatorname{sign}\left(x_{2}\right), \ldots, \operatorname{sign}\left(x_{n}\right)\right)^{T}$ the sign vector of $x \in \mathbb{R}^{n}$, where $\operatorname{sign}(r)$ represents the sign function of $r \in \mathbb{R}$. Obviously, the relations

$$
\|x\|_{1}=\langle x, \operatorname{sign}(x)\rangle, \quad\|x\|_{1} \geq\langle x, \operatorname{sign}(y)\rangle
$$

hold for all $x, y \in \mathbb{R}^{n}$.

In order to discuss the stability of the neural networks (1), we firstly consider exponential stability of the following differential equation with variable coefficients, delays, and impulses

$$
\begin{gathered}
\frac{d z(t)}{d t}=e^{t}\left[F(z(t))+G\left(z_{t}(s)\right)\right], \quad t \geq 0, t \neq t_{k}, \\
\Delta z\left(t_{k}\right)=z\left(t_{k}^{+}\right)-z\left(t_{k}^{-}\right)=\mathscr{I}_{k}\left(z\left(t_{k}\right)\right), \quad k \in \mathbb{N}, \\
z_{0}=\phi \in \mathscr{C}([-\tau, 0], \Omega),
\end{gathered}
$$

where $\tau>0, \mathscr{C}([-\tau, 0], \Omega)$ denotes the space of all continuous functions from $[-\tau, 0]$ into the open subset $\Omega$ of $\mathbb{R}^{n} ; z_{t} \in$ $\mathscr{C}([-\tau, 0], \Omega)$ is defined by $z_{t}(s)=z(t+s)$ for all $s \in[-\tau, 0]$ and $\left\|z_{t}\right\|_{\mathscr{C}}=\sup _{-\tau \leq s \leq 0}\|z(t+s)\|_{1} ; F$ and $G: \Omega \rightarrow \mathbb{R}^{n}$ are nonlinear operators; $0=t_{0}<t_{1}<t_{2}<\cdots$ is a strictly increasing sequence such that $\lim _{k \rightarrow+\infty} t_{k}=+\infty$; $z_{t}(s)$ is defined as follows:

$$
z_{t}(s)=\left(z_{1}(t-s), z_{2}(t-s), \ldots, z_{n}(t-s)\right)^{T} .
$$

The nonlinear operators $F$ and $G$ are defined, respectively, by

$$
\begin{aligned}
F(u) & =\left(F_{1}(u), F_{2}(u), \ldots, F_{n}(u)\right)^{T}, \\
G(u) & =\left(G_{1}(u), G_{2}(u), \ldots, G_{n}(u)\right)^{T} .
\end{aligned}
$$

Definition 1 (see [20]). (1) A nonlinear operator $T: \Omega \rightarrow$ $\mathbb{R}^{n}$ is called be Lipschitz continuous on $\Omega$ if there exists a nonnegative constant $M$ such that

$$
\|T(x)-T(y)\|_{1} \leq M\|x-y\|_{1} \quad \forall x, y \in \Omega,
$$

where $M$ is called the Lipschitz constant of $T$ on $\Omega$. The constant

$$
L_{\Omega}(T)=\sup _{x, y \in \Omega, y \neq x} \frac{\|T(y)-T(x)\|_{1}}{\|y-x\|_{1}}
$$

is called the minimal Lipschitz constant (MLC) of $T$ on $\Omega$. Furthermore, the operator $T$ is called globally Lipschitz continuous if $\Omega=\mathbb{R}^{n}$. 
(2) A nonlinear operator $T: \Omega \rightarrow \mathbb{R}^{n}$ is said to be partially Lipschitz continuous on $\Omega$ if, for any $x \in \Omega$, there exists a constant $L_{x}>0$ such that

$$
\|T(y)-T(x)\|_{1} \leq L_{x}\|y-x\|_{1}, \quad \forall y \in \Omega .
$$

The constant

$$
L_{\Omega}^{p}(T, x)=\sup _{x, y \in \Omega, y \neq x} \frac{\|T(y)-T(x)\|_{1}}{\|y-x\|_{1}}
$$

is called minimal partial Lipschitz constant (MPLC) of $T$ on $\Omega$ with respect to $x$. Furthermore, the operator $T$ is called partially Lipschitz continuous if $\Omega=\mathbb{R}^{n}$.

From the paper [20] we conclude that every Lipschitz continuous operator on $\Omega$ is partially Lipschitz continuous on $\Omega$ and $L_{\Omega}^{p}(T, x) \leq L_{\Omega}(T)$ for any Lipschitz continuous operator $T$ and $x \in \Omega$.

Definition 2 (see [34]). Assume that $\Omega$ is an open subset of $\mathbb{R}^{n}, F$ is a nonlinear operator from $\Omega$ into $\mathbb{R}^{n}$, and $x^{0} \in \Omega$ is any vector. The constant

$$
m_{\Omega}\left(F, x^{0}\right)=\sup _{x \in \Omega, x \neq x^{0}} \frac{\left\langle F(x)-F\left(x^{0}\right), \operatorname{sign}\left(x-x^{0}\right)\right\rangle}{\left\|x-x^{0}\right\|_{1}}
$$

is called relative nonlinear measure of $F$ at $x^{0}$.

Definition 3. $z^{*}$ is said to be an equilibrium point of (15) if $(F+G) z^{*}=0$ and $\mathscr{I}_{k}\left(z^{*}\right)=0$ for all $k \in \mathbb{N}$.

Definition 4. Let $z^{*}$ be an equilibrium point of (15) and $\Omega$ an open neighborhood of $z^{*} . z^{*}$ is exponentially stable on $\Omega$ if there exist two positive constants $\sigma$ and $M$ such that

$$
\left\|z(t)-z^{*}\right\|_{1} \leq M e^{-\sigma t} \max _{-\tau \leq s \leq 0}\left\|\phi(s)-z^{*}\right\|_{1}
$$

holds for $t \geq 0$, where $z(t)$ is the unique solution of (15) initiated from the function $\phi \in \mathscr{C}([-\tau, 0], \Omega)$.

Particularly, if $\Omega=\mathbb{R}^{n}$ holds, then $z^{*}$ is the unique equilibrium point and (15) is said to be globally exponentially stable.

Lemma 5 (see [35]). If $a>c \geq 0$, for every nonnegative real number $b$, the equation

$$
0=\lambda-a+c e^{\lambda b}
$$

has a unique positive solution.

Lemma 6 (see [36]). Let $v(t)>0$ for $t \in \mathbb{R}$ and $t_{0} \in \mathbb{R}$. Suppose that

$$
v^{\prime}(t) \leq-a v(t)+b\left[\sup _{-\infty<s \leq t} v(s)\right] \quad \text { for } t \geq t_{0} .
$$

If $a>b>0$, there exist constants $\gamma>0$ and $k>0$ such that

$$
v(t) \leq k e^{-\gamma\left(t-t_{0}\right)} \sup _{-\infty<s \leq t_{0}} v(s)
$$

holds for $t \geq t_{0}$.
Theorem 7. Let $\Omega$ be an open neighborhood of the equilibrium point $z^{*}$ of (15). Equation (15) has no other equilibrium point in $\Omega$ different from $z^{*}$ if $m_{\Omega}\left(F+G, z^{*}\right)<0$.

Proof. Assume that $\widetilde{z} \in \Omega$ is any equilibrium point of (15) different from $z^{*}$; that is,

$$
\begin{gathered}
F\left(z^{*}\right)+G\left(z^{*}\right)=F(\widetilde{z})+G(\widetilde{z})=0, \\
\mathscr{I}_{k}\left(z^{*}\right)=\mathscr{I}_{k}(\widetilde{z})=0 .
\end{gathered}
$$

Then, we derive

$$
\begin{aligned}
& m_{\Omega}\left(F+G, z^{*}\right) \\
& =\sup _{x \in \Omega, x \neq z^{*}}\left(\left(\left\langleF(x)+G(x)-\left(F\left(z^{*}\right)-G\left(z^{*}\right)\right),\right.\right.\right. \\
& \left.\left.\left.\operatorname{sign}\left(x-z^{*}\right)\right\rangle\right) \times\left(\left\|x-z^{*}\right\|_{1}\right)^{-1}\right) \\
& \geq \frac{\left\langle F(\widetilde{z})+G(\widetilde{z})-\left(F\left(z^{*}\right)+G\left(z^{*}\right)\right), \operatorname{sign}\left(\widetilde{z}-z^{*}\right)\right\rangle}{\left\|\widetilde{z}-z^{*}\right\|_{1}} \\
& =0,
\end{aligned}
$$

which contradicts $m_{\Omega}\left(F+G, z^{*}\right)<0$.

Theorem 8. Let $\Omega$ be a neighborhood of the equilibrium $z^{*}$ of (15). Assume $F$ and $G$ to be partially Lipschitz continuous on $\Omega$ with respect to $z^{*}$ and

$$
\mathscr{I}_{k}\left(z\left(t_{k}\right)\right)=-\gamma_{k}\left(z\left(t_{k}\right)-z^{*}\right), \quad 0 \leq \gamma_{k} \leq 2, k \in \mathbb{N} .
$$

If there exists some diagonal matrix $A=\operatorname{diag}\left(a_{1}, a_{2}, \ldots, a_{n}\right)$ with $a_{i}>0$ such that the inequality

$$
m_{A^{-1}(\Omega)}\left(F A, z^{*}\right)+L_{A^{-1}(\Omega)}^{p}\left(G A, z^{*}\right)<0
$$

holds, then $z^{*}$ is exponentially stable on $\Omega$. Particularly, the solution $z(t)$ of $(15)$ initiated from $\phi \in \mathscr{C}([-\tau, 0], \Omega)$ decays by

$$
\left\|z(t)-z^{*}\right\|_{1} \leq e^{-\sigma t} \cdot \sup _{-\tau \leq s \leq 0}\left\|\phi(s)-z^{*}\right\|_{1} \quad \forall t \geq 0
$$

where $\sigma$ is the unique positive solution of the equation

$$
0=\sigma \cdot \min _{1 \leq i \leq n} a_{i}+m_{A^{-1}(\Omega)}\left(F A, z^{*}\right)+L_{A^{-1}(\Omega)}^{p}\left(G A, z^{*}\right) \cdot e^{\sigma} .
$$

Proof. Let $x(t)=z(t)-z^{*}$ for all $t \geq 0$. From the relations (14) we derive that

$$
\frac{\|x(t)\|_{1}-\|x(t-s)\|_{1}}{s} \leq \frac{1}{s}\langle x(t)-x(t-s), \operatorname{sign}(x(t))\rangle
$$

holds for all $s>0$. Consequently, the function $t \mapsto$ $\|x(t)\|_{1}$ is absolutely continuous in $(0,+\infty)$, which implies that derivatives of $\|x(t)\|_{1}$ exist almost everywhere in $(0,+\infty)$. 
Furthermore, from (15) we conclude that derivatives of $\|x(t)\|_{1}$ satisfy

$$
\begin{aligned}
& \frac{d\|x(t)\|_{1}}{d t} \leq\left\langle\frac{d x(t)}{d t}, \operatorname{sign}(x(t))\right\rangle \\
& =\left\langle e^{t}\left[F(z(t))+G\left(z_{t}(s)\right)\right], \operatorname{sign}(x(t))\right\rangle \\
& =\left\langle\left[ e^{t}\left(F(z(t))+G\left(z_{t}(s)\right)\right)\right.\right. \\
& \left.\left.-\left(F\left(z^{*}\right)+G\left(z^{*}\right)\right)\right], \operatorname{sign}(x(t))\right\rangle \\
& =e^{t}\left[\left\langle F(z(t))-F\left(z^{*}\right), \operatorname{sign}(x(t))\right\rangle\right. \\
& \left.+\left\langle G\left(z_{t}(s)\right)-G\left(z^{*}\right), \operatorname{sign}(x(t))\right\rangle\right] \\
& \leq e^{t}\left[\left\langle F(z(t))-F\left(z^{*}\right), \operatorname{sign}\left(A^{-1} x(t)\right)\right\rangle\right. \\
& \left.+\left\|G\left(z_{t}(s)\right)-G\left(z^{*}\right)\right\|_{1}\right] \\
& \leq e^{t}\left[\left\langle F(z(t))-F\left(z^{*}\right), \operatorname{sign}\left(A^{-1} x(t)\right)\right\rangle\right. \\
& \left.+L_{A^{-1}(\Omega)}^{p}\left(G A, z^{*}\right)\left\|A^{-1} z_{t}(s)-A^{-1} z^{*}\right\|_{1}\right] \\
& \leq e^{t}\left[m_{A^{-1}(\Omega)}\left(F A, z^{*}\right)\left\|A^{-1} x(t)\right\|_{1}+L_{A^{-1}(\Omega)}^{p}\left(G A, z^{*}\right)\right. \\
& \left.\times \sup _{-\tau \leq s \leq 0}\left\|A^{-1}(z(t+s)-y(t+s))\right\|_{1}\right] \\
& \leq e^{t}\left[m_{A^{-1}(\Omega)}\left(F A, z^{*}\right)\|x(t)\|_{1}+L_{A^{-1}(\Omega)}^{p}\left(G A, z^{*}\right)\right. \\
& \left.\times \sup _{-\tau \leq s \leq t}\|x(s)\|_{1}\right]\left(\min _{1 \leq i \leq n} a_{i}\right)^{-1} \\
& \leq e^{t_{k}}\left[m_{A^{-1}(\Omega)}\left(F A, z^{*}\right)\|x(t)\|_{1}+L_{A^{-1}(\Omega)}^{p}\left(G A, z^{*}\right)\right. \\
& \left.\times \sup _{-\tau \leq s \leq t}\|x(s)\|_{1}\right]\left(\min _{1 \leq i \leq n} a_{i}\right)^{-1}, \quad t \in\left(t_{k-1}, t_{k}\right) .
\end{aligned}
$$

The combination of condition (30) and Lemmas 6 and 5 implies that

$$
\|x(t)\|_{1} \leq e^{-\sigma_{t_{k}} t} \sup _{-\tau \leq s \leq 0}\|x(s)\|_{1}
$$

holds for all $t \in\left(t_{k-1}, t_{k}\right)$, where $\sigma_{t_{k}}$ is the unique positive solution of the equation

$$
\begin{aligned}
0= & e^{-t_{k}} \cdot \sigma \cdot \min _{1 \leq i \leq n} a_{i}+m_{A^{-1}(\Omega)}\left(F A, z^{*}\right) \\
& +L_{A^{-1}(\Omega)}^{p}\left(G A, z^{*}\right) \cdot e^{\sigma} .
\end{aligned}
$$

It needs to point out that the positive solution $\sigma_{t_{k}}$ of (36) is strictly monotonically increasing with respect to $t_{k}$. In fact, let $\sigma_{t_{k+1}}$ be the positive solution of the equation

$$
\begin{aligned}
0= & e^{-t_{k+1}} \cdot \sigma \cdot \min _{1 \leq i \leq n} a_{i}+m_{A^{-1}(\Omega)}\left(F A, z^{*}\right) \\
& +L_{A^{-1}(\Omega)}^{p}\left(G A, z^{*}\right) \cdot e^{\sigma} .
\end{aligned}
$$

By subtracting (37) from (36), we derive

$$
\begin{aligned}
& \left(e^{-t_{k}} \sigma_{t_{k}}-e^{-t_{k+1}} \sigma_{t_{k+1}}\right) \min _{1 \leq i \leq n} a_{i} \\
& +L_{A^{-1}(\Omega)}^{p}\left(G A, z^{*}\right)\left(e^{\sigma_{t_{k}}}-e^{\sigma_{t_{k+1}}}\right)=0 .
\end{aligned}
$$

Furthermore, we have

$$
e^{\sigma_{t_{k+1}}}-e^{\sigma_{t_{k}}}=\frac{\min _{1 \leq i \leq n} a_{i}}{L_{A^{-1}(\Omega)}^{p}\left(G A, z^{*}\right)}\left(e^{-t_{k}} \sigma_{t_{k}}-e^{-t_{k+1}} \sigma_{t_{k+1}}\right) .
$$

Since both of $\min _{1 \leq i \leq n} a_{i}$ and $L_{A^{-1}(\Omega)}\left(G A, z^{*}\right)$ are positive, we have

$$
\left(e^{\sigma_{t_{k+1}}}-e^{\sigma_{t_{k}}}\right)\left(e^{-t_{k}} \sigma_{t_{k}}-e^{-t_{k+1}} \sigma_{t_{k+1}}\right)>0 .
$$

It is obvious that $\sigma_{t_{k}}$ is not equal to $\sigma_{t_{k+1}}$, that is, $\sigma_{t_{k+1}}<\sigma_{t_{k}}$ or $\sigma_{t_{k+1}}>\sigma_{t_{k}}$. If $\sigma_{t_{k+1}}<\sigma_{t_{k}}$, then $e^{\sigma_{t_{k+1}}}<e^{\sigma_{t_{k}}}$ and $e^{-t_{k}} \sigma_{t_{k}}-$ $e^{-t_{k+1}} \sigma_{t_{k+1}}<0$ from inequality (40), that is,

$$
\frac{\sigma_{t_{k+1}}}{\sigma_{t_{k}}}>e^{\left(t_{k+1}-t_{k}\right)}
$$

Since $t_{k+1}>t_{k}, \sigma_{t_{k+1}} / \sigma_{t_{k}}>e^{\left(t_{k+1}-t_{k}\right)}>1$ contradict the assumption $\sigma_{t_{k+1}}<\sigma_{t_{k}}$. This means that $\sigma_{t_{k+1}}>\sigma_{t_{k}}$ holds for $t_{k+1}>t_{k}$; that is, the positive solution $\sigma_{t_{k}}$ of (36) is strictly monotonically increasing with respect to $t_{k}$. Hence, $\sigma=\sigma_{t_{0}}<\sigma_{t_{1}}<\sigma_{t_{2}}<\cdots$, where $\sigma_{t_{0}}$ is the unique positive solution of (37) at $t_{0}=0$; that is,

$$
0=\sigma \cdot \min _{1 \leq i \leq n} a_{i}+m_{A^{-1}(\Omega)}\left(F A, z^{*}\right)+L_{A^{-1}(\Omega)}^{p}\left(G A, z^{*}\right) \cdot e^{\sigma} .
$$

Since $e^{-\sigma_{t_{k}} t}<e^{-\sigma_{t_{0}} t}=e^{-\sigma t}$ for all $t \in\left(t_{k-1}, t_{k}\right), k \in \mathbb{N}$, inequality (35) means that

$$
\|x(t)\|_{1} \leq e^{-\sigma t} \sup _{-\tau \leq s \leq 0}\|x(s)\|_{1}
$$

holds for all $t \in\left(t_{k-1}, t_{k}\right), k \in \mathbb{N}$, where $\sigma$ is the unique positive solution of (32). Inequality (43) is accordingly changed into the following form:

$$
\left\|z(t)-z^{*}\right\|_{1} \leq e^{-\sigma t} \sup _{-\tau \leq s \leq 0}\left\|\phi(s)-z^{*}\right\|_{1}
$$

which holds for all $t \in\left(t_{k-1}, t_{k}\right), k \in \mathbb{N}$. According to condition (29), we enjoy

$$
\begin{aligned}
x\left(t_{k}^{+}\right) & =z\left(t_{k}^{+}\right)-z^{*}=z\left(t_{k}\right)+\mathscr{I}_{k}\left(z\left(t_{k}\right)\right)-z^{*} \\
& =\left(1-\gamma_{k}\right) x\left(t_{k}\right), \quad k \in \mathbb{N} .
\end{aligned}
$$


This implies

$$
\left\|x\left(t_{k}^{+}\right)\right\|_{1}=\left\|\left(1-\gamma_{k}\right) x\left(t_{k}\right)\right\|_{1} \leq\left\|x\left(t_{k}\right)\right\|_{1}, \quad k \in \mathbb{N} \text {. }
$$

From (35) and (46) we derive

$$
\begin{aligned}
\left\|z\left(t_{k}^{+}\right)-z^{*}\right\|_{1} & =\left\|x\left(t_{k}^{+}\right)\right\|_{1} \leq\left\|x\left(t_{k}\right)\right\|_{1}=\left\|x\left(t_{k}^{-}\right)\right\|_{1} \\
& \leq e^{-\sigma t} \sup _{-\tau \leq s \leq 0}\|x(s)\|_{1} \\
& =e^{-\sigma t} \sup _{-\tau \leq s \leq 0}\left\|\phi(s)-z^{*}\right\|_{1}, \quad k \in \mathbb{N} .
\end{aligned}
$$

In conclusion, we obtain inequality (31).

Remark 9. Our proof idea mainly comes from Theorem 2 of [20] investigating the exponential stability of the special case of (15) (i.e., (15) with constant coefficients). However, they are essentially different because Theorem 8 in this paper has to deal with time-varying coefficients. Consequently, Theorem 8 in this paper is a generalization of Theorem 2 in [20]. Moreover, it needs to point out that the exponential stability criterion (30) and exponential decay index $\sigma$ in (31) are independent of time $t$ although the abstract equation (15) enjoys time-varying coefficients, which means that our method is essential to qualitatively and quantitatively characterize exponential stability of (15). Moreover, Theorem 8 is not only generalization and improvement of Theorem 1 in [35] because there indeed exists a nonlinear Lipschitz continuous map $T$ on $\Omega$ such that $L_{\Omega}^{p}(T, x)$ is strictly less than $L_{\Omega}(T)$ for any $x \in \Omega$ and (15) enjoys time-varying coefficients.

It is obvious that the CNNs model (12) can be changed into the form of (15). By Theorem 8, we can obtain the exponential stable criterion of equilibrium point of the CNNs model (12). Since the model (1) is equivalent to the model (12) in the sense of solution, models (12) and (1) enjoy the same equilibrium point $v^{*}=u^{*}$, where $v^{*}=\left(v_{1}^{*}, v_{2}^{*}, \ldots, v_{n}^{*}\right)^{T}$ and $u^{*}=\left(u_{1}^{*}, u_{2}^{*}, \ldots, u_{n}^{*}\right)^{T}$ are the equilibrium point of models (12) and (1), respectively. What qualitative property of the model (1) can be derived from the global exponential stability of the model (12)? The next theorem can answer this problem.

Theorem 10. Suppose that the equilibrium point $u^{*}$ of the model (12) is globally exponentially stable, that is, that exist two positive constants $M$ and $\sigma$ such that

$$
\left\|v(t)-u^{*}\right\|_{1} \leq M e^{\sigma t} \max _{-\tau \leq s \leq 0}\left\|\psi(s)-u^{*}\right\|_{1}
$$

holds for $t \geq 0$, where $v(t)$ is the unique solution of the model (12) initiated from $\psi \in \mathscr{C}\left([-\tau, 0], \mathbb{R}^{n}\right)$. Then $u^{*}$ of the model (1) is globally asymptotic stable. Particularly, the inequality

$$
\left\|u(t)-u^{*}\right\|_{1} \leq M t^{-\sigma} \max _{r \leq s \leq 1}\left\|\phi(s)-u^{*}\right\|_{1}
$$

holds for $t \geq 1$, where $u(t)$ is the unique solution of the model (1) initiated from $\phi \in \mathscr{C}\left([r, 1], \mathbb{R}^{n}\right), r=\min _{1 \leq j \leq n}\left\{p_{j}, q_{j}\right\}$.
Proof. By the transformation (2) and the inequality (48), we derive

$$
\begin{aligned}
\left\|u\left(e^{t}\right)-u^{*}\right\|_{1} & =\left\|v(t)-u^{*}\right\|_{1} \\
& \leq M e^{-\sigma t} \max _{-\tau \leq s \leq 0}\left\|\psi(s)-u^{*}\right\|_{1} \\
& =M e^{-\sigma t} \max _{-\tau \leq s \leq 0}\left\|\phi\left(e^{s}\right)-u^{*}\right\|_{1}, \\
& e^{t} \geq 1 .
\end{aligned}
$$

Let $e^{t}=h$, then $h \geq 1$ and $t=\ln h \geq 0$. Let $e^{s}=\xi$, then $\xi \in[r, 1]$. The inequality (50) implies

$$
\begin{aligned}
\left\|u(h)-u^{*}\right\| & \leq M e^{-\sigma \ln h} \max _{r \leq \xi \leq 1}\left\|\phi(\xi)-u^{*}\right\|_{1} \\
& =M h^{-\sigma} \max _{r \leq \xi \leq 1}\left\|\phi(\xi)-u^{*}\right\|_{1}, \quad h \geq 1 .
\end{aligned}
$$

Taking $t=h$, we furthermore derive

$$
\left\|u(t)-u^{*}\right\| \leq M t^{-\sigma} \max _{r \leq \xi \leq 1}\left\|\phi(\xi)-u^{*}\right\|_{1}, \quad t \geq 1 .
$$

This implies that the equilibrium point $u^{*}$ of the model (1) is globally asymptotic stable.

Remark 11. It need point out that the paper [7] has obtained not exponential stability, but asymptotic stable criteria of CNNs with multi-proportional delays because it mistakes asymptotic stability as exponential stability, which can be easily seen from the Remark 3.2 in [7] and Theorem 10 in this paper.

\section{Uniqueness and Global Asymptotic Stability of Equilibrium Point of Model (1)}

In this subsection, we firstly prove that model (1) has a unique equilibrium point in $\mathbb{R}^{n}$. It is enough to prove that model (12) has a unique equilibrium point in $\mathbb{R}^{n}$ because models (12) and (1) enjoy the same equilibrium point. For this, we define that $F=\left(F_{1}, F_{2}, \ldots, F_{n}\right)^{T}$ and $G=\left(G_{1}, G_{2}, \ldots, G_{n}\right)^{T}: \mathbb{R}^{n} \rightarrow \mathbb{R}^{n}$ are defined, respectively, by

$$
\begin{gathered}
F_{i}(v)=-d_{i} v_{i}+\sum_{j=1}^{n} a_{i j} f_{j}\left(v_{j}\right), \\
G_{i}(v)=\sum_{j=1}^{n}\left[b_{i j} g_{j}\left(v_{j}\right)+c_{i j} h_{j}\left(v_{j}\right)\right]+I_{i} .
\end{gathered}
$$

Theorem 12. Suppose that the assumption $(H)$ holds and $u^{*}$ is an equilibrium point of the model (1). For each set of external inputs, $I_{i}$, model (1) has no other equilibrium point in $\mathbb{R}^{n}$ different from $u^{*}$ if there exist positive real numbers $a_{i}(i=1,2$, $\ldots, n)$ such that

$$
\begin{gathered}
\max _{1 \leq j \leq n} \frac{1}{d_{j}} \sum_{i=1}^{n}\left[L_{\mathbb{R}_{j}}^{p}\left(f_{j}, u_{j}^{*}\right) \frac{a_{j}}{a_{i}}\left|a_{i j}\right|+L_{\mathbb{R}_{j}}^{p}\left(g_{j}, u_{j}^{*}\right) \frac{a_{j}}{a_{i}}\left|b_{i j}\right|\right. \\
\left.+L_{\mathbb{R}_{j}}^{p}\left(h_{j}, u_{j}^{*}\right) \frac{a_{j}}{a_{i}}\left|c_{i j}\right|\right]<1
\end{gathered}
$$


holds, where $L_{\mathbb{R}_{j}}^{p}\left(f_{j}, u_{j}^{*}\right), L_{\mathbb{R}_{j}}^{p}\left(g_{j}, u_{j}^{*}\right)$, and $L_{\mathbb{R}_{j}}^{p}\left(h_{j}, u_{j}^{*}\right)$ denote MPLC of $f_{j}, g_{j}$, and $h_{j}$ on $\mathbb{R}_{j}$ with respect to $u_{j}^{*}$, respectively.

Proof. Obviously, it is enough to prove that model (12) has no other equilibrium point in $\mathbb{R}^{n}$ different from $u^{*}$ if the inequality (54) holds. Define $A=\operatorname{diag}\left(a_{1}, a_{2}, \ldots, a_{n}\right)$ and we need only prove $m_{A^{-1}\left(\mathbb{R}^{n}\right)}\left(A^{-1}(F+G) A, u^{*}\right)<0$ according to Theorem 7. In detail, for $v \in A^{-1}\left(\mathbb{R}^{n}\right)$, we enjoy

$$
\begin{aligned}
& \left\langle A^{-1}(F+G)(A v)-A^{-1}(F+G)\left(A u^{*}\right), \operatorname{sign}\left(v-u^{*}\right)\right\rangle \\
& =\sum_{i=1}^{n} \operatorname{sign}\left(v_{i}-u_{i}^{*}\right) \\
& \times\left\{-a_{i}^{-1}\left[d_{i}\left(a_{i} v_{i}\right)-d_{i}\left(a_{i} u_{i}^{*}\right)\right]\right. \\
& +\sum_{j=1}^{n}\left[a_{i}^{-1} a_{i j}\left(f_{j}\left(a_{j} v_{j}\right)-f_{j}\left(a_{j} u_{j}^{*}\right)\right)\right. \\
& +a_{i}^{-1} b_{i j}\left(g_{j}\left(a_{j} v_{j}\right)-g_{j}\left(a_{j} u_{j}^{*}\right)\right) \\
& \left.\left.+a_{i}^{-1} c_{i j}\left(h_{j}\left(a_{j} v_{j}\right)-h_{j}\left(a_{j} u_{j}^{*}\right)\right)\right]\right\} \\
& \leq \sum_{i=1}^{n} a_{i}^{-1}\left\{-\left|d_{i}\left(a_{i} v_{i}\right)-d_{i}\left(a_{i} u_{i}^{*}\right)\right|\right. \\
& +\sum_{j=1}^{n}\left[\left|a_{i j}\right|\left|f_{j}\left(a_{j} v_{j}\right)-f_{j}\left(a_{j} u_{j}^{*}\right)\right|\right. \\
& +\left|b_{i j}\right|\left|g_{j}\left(a_{j} v_{j}\right)-g_{j}\left(a_{j} u_{j}^{*}\right)\right| \\
& \left.\left.+\left|c_{i j}\right|\left|h_{j}\left(a_{j} v_{j}\right)-h_{j}\left(a_{j} u_{j}^{*}\right)\right|\right]\right\} \\
& \leq-\sum_{i=1}^{n} d_{i}\left|v_{i}-u_{i}^{*}\right| \\
& +\sum_{j=1}^{n} \sum_{i=1}^{n} \frac{a_{j}}{a_{i}}\left[\left|a_{i j}\right| L_{\mathbb{R}_{j}}^{p}\left(f_{j}, u_{j}^{*}\right)+\left|b_{i j}\right| L_{\mathbb{R}_{j}}^{p}\left(g_{j}, u_{j}^{*}\right)\right. \\
& \left.+\left|c_{i j}\right| L_{\mathbb{R}_{j}}^{p}\left(h_{j}, u_{j}^{*}\right)\right]\left|v_{j}-u_{j}^{*}\right| \\
& =-\sum_{j=1}^{n}\left\{d_{j}-\sum_{i=1}^{n} \frac{a_{j}}{a_{i}}\left[\left|a_{i j}\right| L_{\mathbb{R}_{j}}^{p}\left(f_{j}, u_{j}^{*}\right)+\left|b_{i j}\right| L_{\mathbb{R}_{j}}^{p}\left(g_{j}, u_{j}^{*}\right)\right.\right. \\
& \left.\left.+\left|c_{i j}\right| L_{\mathbb{R}_{j}}^{p}\left(h_{j}, u_{j}^{*}\right)\right]\right\}\left|v_{j}-u_{j}^{*}\right|
\end{aligned}
$$

The combination of (55) and (54) implies that $m_{A^{-1}\left(\mathbb{R}^{n}\right)}\left(A^{-1}(F+G) A, u^{*}\right)<0$, which implies that model
(12) enjoys no other equilibrium point in $\mathbb{R}^{n}$ different from $u^{*}$. That is to say, $u^{*}$ is the unique equilibrium point in $\mathbb{R}^{n}$ of model (1).

Secondly, we prove that condition (54) also guarantees global asymptotic stability of equilibrium point of model (1) by Theorems 8 and 10 .

Theorem 13. Assume that assumption $(H)$ holds, $u^{*}$ is the equilibrium point of the model (1), and $\mathscr{I}_{i, k}\left(u_{i}\left(t_{k}\right)\right)=$ $-\gamma_{i, k}\left(u_{i}\left(t_{k}\right)-u_{i}^{*}\right), 0 \leq \gamma_{i, k} \leq 2$, for $k \in \mathbb{N}$ and $i=1,2, \ldots, n$. If there exist a set of positive real numbers $a_{i}(i=1,2, \ldots, n)$ such that condition (54) holds, then for each set of external input, $I_{i}$, model (1) is globally asymptotic stable. Particularly, if $u(t)$ is the solution of the model (1) initiated from $\phi \in \mathscr{C}\left([r, 1], \mathbb{R}^{n}\right)$, then the inequality

$$
\left\|u(t)-u^{*}\right\|_{1} \leq t^{-\sigma} \cdot \frac{\max _{1 \leq i \leq n} a_{i}}{\min _{1 \leq i \leq n} a_{i}} \cdot \sup _{r \leq s \leq 1}\left\|\phi(s)-u^{*}\right\|_{1}
$$

holds for $t \geq 1$, where $\sigma$ is the unique positive solution of the equation

$$
\sigma \cdot \min _{1 \leq j \leq n} c_{j}^{-1}-1+k e^{\sigma}=0
$$

with

$$
\begin{gathered}
c_{j}=d_{j}-L_{\mathbb{R}_{j}}^{p}\left(f_{j}, u_{j}^{*}\right) \sum_{i=1}^{n} \frac{a_{j}}{a_{i}}\left|a_{i j}\right|, \\
k=\max _{1 \leq j \leq n}\left\{c_{j}^{-1} \sum_{i=1}^{n} \frac{a_{j}}{a_{i}}\left[\left|b_{i j}\right| L_{\mathbb{R}_{j}}^{p}\left(g_{j}, u_{j}^{*}\right)+\left|c_{i j}\right| L_{\mathbb{R}_{j}}^{p}\left(h_{j}, u_{j}^{*}\right)\right]\right\} .
\end{gathered}
$$

Proof. Obviously, $u^{*}$ is also the equilibrium point of model (12) because model (1) is equivalent to the model (12) in the sense of solution. Firstly, we prove that model (12) is globally exponentially stable by Theorem 8 . For this, let $A=\operatorname{diag}\left(a_{1}, a_{2}, \ldots, a_{n}\right)$ and $C=\operatorname{diag}\left(c_{1}^{-1}, c_{2}^{-1}, \ldots, c_{n}^{-1}\right)$. It immediately follows from the condition (54) that

$$
c_{j}=d_{j}-L_{\mathbb{R}_{j}}^{p}\left(f_{j}, u_{j}^{*}\right) \sum_{i=1}^{n} \frac{a_{j}}{a_{i}}\left|a_{i j}\right|>0, \quad \text { for } j=1,2, \ldots, n \text {. }
$$

For all $v \in C^{-1} A^{-1}\left(\mathbb{R}^{n}\right)$,

$$
\begin{aligned}
\left\langle A^{-1} F(A C v)-A^{-1} F\left(A C u^{*}\right), \operatorname{sign}\left(v-u^{*}\right)\right\rangle \\
\leq \sum_{i=1}^{n} a_{i}^{-1}\left\{-\left|d_{i}\left(a_{i} c_{i}^{-1} v_{i}\right)-d_{i}\left(a_{i} c_{i}^{-1} u_{i}^{*}\right)\right|\right. \\
\left.+\sum_{j=1}^{n}\left|a_{i j}\right|\left|f_{j}\left(a_{j} c_{j}^{-1} v_{j}\right)-f_{j}\left(a_{j} c_{j}^{-1} u_{j}^{*}\right)\right|\right\}
\end{aligned}
$$




$$
\begin{aligned}
& \leq \sum_{i=1}^{n} a_{i}^{-1}\left\{-a_{i} c_{i}^{-1} d_{i}\left|v_{i}-u_{i}^{*}\right|\right. \\
& \left.+\sum_{j=1}^{n}\left|a_{i j}\right| L_{\mathbb{R}_{j}}^{p}\left(f_{j}, u_{j}^{*}\right) a_{j} c_{j}^{-1}\left|v_{j}-u_{j}^{*}\right|\right\} \\
& =-\sum_{j=1}^{n} c_{j}^{-1}\left(d_{j}-L_{\mathbb{R}_{j}}^{p}\left(f_{j}, u_{j}^{*}\right) \sum_{i=1}^{n} \frac{a_{j}}{a_{i}}\left|a_{i j}\right|\right)\left|v_{j}-u_{j}^{*}\right| \\
& =-\left\|v-u^{*}\right\|_{1} \text {, }
\end{aligned}
$$

which implies that $m_{C^{-1} A^{-1}\left(\mathbb{R}^{n}\right)}\left(A^{-1} F A C, u^{*}\right) \leq-1$. For all $v \in$ $C^{-1} A^{-1}\left(\mathbb{R}^{n}\right)$, we have

$$
\begin{gathered}
\left\|A^{-1} G A C v-A^{-1} G A C u^{*}\right\|_{1} \\
=\sum_{i=1}^{n} \mid a_{i}^{-1} \sum_{j=1}^{n}\left\{b_{i j}\left[g_{j}\left(a_{j} c_{j}^{-1} v_{j}\right)-g_{j}\left(a_{j} c_{j}^{-1} u_{j}^{*}\right)\right]\right. \\
\left.+c_{i j}\left[h_{j}\left(a_{j} c_{j}^{-1} v_{j}\right)-h_{j}\left(a_{j} c_{j}^{-1} u_{j}^{*}\right)\right]\right\} \mid \\
\leq \sum_{j=1}^{n}\left\{c _ { j } ^ { - 1 } \sum _ { i = 1 } ^ { n } \frac { a _ { j } } { a _ { i } } \left[\left|b_{i j}\right| L_{\mathbb{R}_{j}}^{p}\left(g_{j}, u_{j}^{*}\right)\right.\right. \\
\left.\left.+\left|c_{i j}\right| L_{\mathbb{R}_{j}}^{p}\left(h_{j}, u_{j}^{*}\right)\right]\right\}\left|v_{j}-u_{j}^{*}\right| ;
\end{gathered}
$$

thus,

$$
\begin{aligned}
& L_{C^{-1} A^{-1}\left(\mathbb{R}^{n}\right)}^{p}\left(A^{-1} G A C, u^{*}\right) \\
& \quad \leq \max _{1 \leq j \leq n}\left\{c_{j}^{-1} \sum_{i=1}^{n} \frac{a_{j}}{a_{i}}\left[\left|b_{i j}\right| L_{\mathbb{R}_{j}}^{p}\left(g_{j}, u_{j}^{*}\right)+\left|c_{i j}\right| L_{\mathbb{R}_{j}}^{p}\left(h_{j}, u_{j}^{*}\right)\right]\right\} \\
& \quad=k .
\end{aligned}
$$

Consequently, from (54) we conclude that

$$
\begin{gathered}
m_{C^{-1} A^{-1}\left(\mathbb{R}^{n}\right)}\left(A^{-1} F A C, u^{*}\right)+L_{C^{-1} A^{-1}\left(\mathbb{R}^{n}\right)}^{p}\left(A^{-1} G A C, u^{*}\right) \\
\leq-1+\max _{1 \leq j \leq n}\left\{c _ { j } ^ { - 1 } \sum _ { i = 1 } ^ { n } \frac { a _ { j } } { a _ { i } } \left[\left|b_{i j}\right| L_{\mathbb{R}_{j}}^{p}\left(g_{j}, u_{j}^{*}\right)\right.\right. \\
\left.\left.+\left|c_{i j}\right| L_{\mathbb{R}_{j}}^{p}\left(h_{j}, u_{j}^{*}\right)\right]\right\} \\
=\max _{1 \leq j \leq n}\left\{\left(-d_{j}+\sum_{i=1}^{n} \frac{a_{j}}{a_{i}}\left|a_{i j}\right| L_{\mathbb{R}_{j}}^{p}\left(f_{j}, u_{j}^{*}\right)\right.\right.
\end{gathered}
$$

$$
\begin{aligned}
& \left.+\sum_{i=1}^{n} \frac{a_{j}}{a_{i}}\left[\left|b_{i j}\right| L_{\mathbb{R}_{j}}^{p}\left(g_{j}, u_{j}^{*}\right)+\left|c_{i j}\right| L_{\mathbb{R}_{j}}^{p}\left(h_{j}, u_{j}^{*}\right)\right]\right) \\
& \left.\times\left(d_{j}-\sum_{i=1}^{n} \frac{a_{j}}{a_{i}}\left|a_{i j}\right| L_{\mathbb{R}_{j}}^{p}\left(f_{j}, u_{j}^{*}\right)\right)^{-1}\right\}<0 .
\end{aligned}
$$

By Theorem 8 , the solution $y(t)$ of the functional differential equation

$$
\begin{gathered}
\frac{d y(t)}{d t}=e^{t}\left[A^{-1} F A C(y(t))+A^{-1} G A C\left(y_{t}(s)\right)\right], \\
t \geq 0, \quad t \neq t_{k}, \\
\Delta y\left(t_{k}\right)=y\left(t_{k}^{+}\right)-y\left(t_{k}^{-}\right)=\mathscr{I}_{k}\left(y\left(t_{k}\right)\right), \quad k \in \mathbb{N}, \\
y_{0}=\tilde{\phi} \in \mathscr{C}\left([-\tau, 0], C^{-1} A^{-1}\left(\mathbb{R}^{n}\right)\right),
\end{gathered}
$$

satisfies

$$
\left\|y(t)-C^{-1} A^{-1} u^{*}\right\| \leq e^{-\sigma t} \cdot \sup _{-\tau \leq s \leq 0}\left\|\widetilde{\phi}(s)-C^{-1} A^{-1} u^{*}\right\|_{1}
$$

$\forall t \geq 0$

where $\sigma$ is the unique positive solution of (57). It is obvious that $y(t)=C^{-1} A^{-1} v(t)$ is the solution of $(64)$ if $v(t)$ is a solution of model (12). Consequently, the equilibrium point $u^{*}$ of model (12) is globally exponentially stable; that is,

$$
\left\|v(t)-u^{*}\right\|_{1} \leq e^{\sigma t} \cdot \frac{\max _{1 \leq i \leq n} a_{i}}{\min _{1 \leq i \leq n} a_{i}} \cdot \max _{-\tau \leq s \leq 0}\left\|\psi(s)-u^{*}\right\|_{1}
$$

holds for $t \geq 0$, where $\sigma$ is unique positive solution of (57), $\tau=\max _{1 \leq j \leq n}\left\{\tau_{j}, \varsigma_{j}\right\}, \tau_{j}=-\ln p_{j}>0, \varsigma_{j}=-\ln q_{j}>0$, and $\psi_{i} \in C([-\tau, 0], \mathbb{R})$. By Theorem 10 , we derive that the solution $u(t)$ of model (1) initiated from $\phi \in \mathscr{C}\left([r, 1], \mathbb{R}^{n}\right)$ satisfies inequality (56) for $t \geq 1$. That is to say, the solution of model (1) is globally asymptotically stable.

Remark 14. Theorems 12 and 13 provide a sufficient condition (54) to the uniqueness and global asymptotic stability of the equilibrium point of impulsive CNNs (1) with multiproportional delays and partially Lipschitz continuous activation functions. On the one hand, the proportional delay is time varying, unbounded, and monotonic and the model (1) does not require the proportional delays to meet any other condition. Hence, compared with the results in papers [11-23] with the constant, bounded time varying, or unbounded distributed delays, our results are new. Moreover, the stability of CNNs with general unbounded time varying delays in [37, 38] and proportional delays in [7-10] has been investigated. Compared with these results, our results are their generalizations because model (1) has impulsive perturbations. On the other hand, model (1) only requires activation functions to be partially Lipschitz continuous. In fact, partial Lipschitz continuity is less conservative; that is, it 
does not meet conventional assumptions, such as, boundedness, global Lipschitz continuity, or monotonicity. Compared with these excellent results on neural networks with globally Lipschitz continuous [25-30], bounded and monotonic [31], or bounded $[24,32]$ activation functions, our results are new. Furthermore, our results are also generalizations of the papers $[24,29,35]$ and even improvement of the paper [35] with globally Lipschitz continuous activation functions and there indeed exists a nonlinear globally Lipschitz continuous map $T$ on $\Omega$ such that $L_{\Omega}^{p}(T, x)$ is strictly less than $L_{\Omega}(T)$ for any $x \in \Omega$.

\section{Illustrative Example}

In this section, we present an illustrative example to verify effectiveness of our method.

Example 1. Consider impulsive CNNs with proportional delays and partially Lipschitz continuous activation functions

$$
\begin{gathered}
\dot{u}(t)=-D u(t)+A f(u(t))+B g(u(p t)) \\
+C h(u(q t))+I, \quad t \geq 1, \quad t \neq t_{k}, \\
\Delta u\left(t_{k}\right)=\mathscr{I}_{k}\left(u\left(t_{k}\right)\right), \quad t=t_{k}, \quad k \in \mathbb{N}, \\
u(s)=\phi(s), \quad r \leq s \leq 1,
\end{gathered}
$$

where

$$
\begin{gathered}
D=\left(\begin{array}{ccc}
5 & 0 & 0 \\
0 & 8 & 0 \\
0 & 0 & 10
\end{array}\right), \quad A=\left(\begin{array}{ccc}
-0.8 & 0.2 & 0.5 \\
-0.7 & -1.3 & 0.6 \\
1.5 & -0.4 & 2.1
\end{array}\right), \\
B=\left(\begin{array}{ccc}
-0.3 & 0.5 & -0.9 \\
1.4 & -0.8 & 1.2 \\
0.2 & -0.1 & -0.5
\end{array}\right), \\
C=\left(\begin{array}{ccc}
-1 & 0.6 & 1.3 \\
0.4 & -1.6 & -0.7 \\
-1.7 & -0.3 & 0.6
\end{array}\right) \\
I=\left(\begin{array}{l}
0 \\
0 \\
0
\end{array}\right), \quad \mathscr{I}_{k}=\left(\begin{array}{l}
-\gamma_{1 k} \\
-\gamma_{2 k} \\
-\gamma_{3 k}
\end{array}\right), \\
P=\left(\begin{array}{l}
0.7 \\
0.5 \\
0.3
\end{array}\right), \quad q=\left(\begin{array}{c}
0.2 \\
0.9 \\
0.5
\end{array}\right),
\end{gathered}
$$

$f_{j}(x)=g_{j}(x)=h_{j}(x)=f(x)=0.5 x\left(\sin ^{2} x+\cos x\right)$ for $x \in \mathbb{R}$ and $j=1,2,3, \Delta u_{1}\left(t_{k}\right)=-\gamma_{1 k} u_{1}\left(t_{k}\right), \Delta u_{2}\left(t_{k}\right)=$ $-\gamma_{2 k} u_{2}\left(t_{k}\right)$, and $\Delta u_{3}\left(t_{k}\right)=-\gamma_{3 k} u_{3}\left(t_{k}\right), t_{1}<t_{2}<\cdots$, is a strictly increasing sequence such that $\lim _{k \rightarrow+\infty} t_{k}=+\infty$ and $\gamma_{1 k}=1+0.9 \sin ^{2}\left(2 k^{2}+k\right), \gamma_{2 k}=1.8+0.2 \sin \left(1+k^{2}\right)$, and $\gamma_{3 k}=1.4+0.5 \cos ^{2}\left(2+3 k^{2}\right), r=\min _{1 \leq j \leq 3}\left\{p_{j}, q_{j}\right\}=0.2$. $\phi_{1}(s)=5+s^{2}, \phi_{2}(s)=-2+s^{3}$ and $\phi_{3}(s)=-4+s$ for $r \leq s \leq 1$.
From the definition of $f(x)$ we can conclude

$$
\begin{aligned}
|f(y)-f(x)| & =\left|y\left(\sin ^{2} y+\cos y\right)-x\left(\sin ^{2} x+\cos x\right)\right| \\
& \leq 0.5(2+3|x|)|y-x|
\end{aligned}
$$

holds for $x, y \in \mathbb{R}$, which means that $f$ is partially Lipschitz continuous on $\mathbb{R}$. It is easily verified that $u^{*}=(0,0,0)^{T}$ is the equilibrium point of the model $(67)$ and $L_{\mathbb{R}}^{p}(f, 0)=0.625$; that is, $L_{\mathbb{R}_{j}}^{p}\left(f_{j}, 0\right)=L_{\mathbb{R}_{j}}^{p}\left(g_{j}, 0\right)=L_{\mathbb{R}_{j}}^{p}\left(h_{j}, 0\right)=0.625$ for $j=$ $1,2,3$. Consequently, the criteria of the papers [24,29] are not applied to this the model (67) because they require activation functions to be globally Lipschitz continuous. Moreover, none of the stability criteria in [11-23] is applied to model (67) since the delay factors $\left(1-p_{j}\right) t$ and $\left(1-q_{j}\right) t$ are unbounded and monotonic and not required to meet any other additional conditions. Although the papers [7-10] have investigated the stability of CNNs with proportional delays, these methods are not able to apply to model (67) because model (67) enjoys the impulsive perturbations.

However, taking $a_{1}=3, a_{2}=6$ and $a_{3}=5$, we obtain

$$
\begin{gathered}
\max _{1 \leq j \leq 3} \frac{1}{d_{j}} \sum_{i=1}^{3}\left\{L_{\mathbb{R}_{j}}^{p}\left(f_{j}, 0\right) \frac{a_{j}}{a_{i}}\left|a_{i j}\right|+L_{\mathbb{R}_{j}}^{p}\left(g_{j}, 0\right) \frac{a_{j}}{a_{i}}\left|b_{i j}\right|\right. \\
\left.+L_{\mathbb{R}_{j}}^{p}\left(h_{j}, 0\right) \frac{a_{j}}{a_{i}}\left|c_{i j}\right|\right\} \\
=\max \{0.6738,0.5672,0.6115\}=0.6738<1,
\end{gathered}
$$

that is, the condition (54) holds for $a_{1}=3, a_{2}=6$, and $a_{3}=5$. Moreover, $0 \leq \gamma_{i k} \leq 2$ for $i=1,2,3$. According to Theorems 10 and 12 , we conclude that the equilibrium point $u^{*}=$ $(0,0,0)^{T}$ of the model $(67)$ is globally asymptotically stable and the solution satisfies the following inequality (Figure 1):

$$
\begin{aligned}
\|u(t)\|_{1} & =\left|u_{1}(t)\right|+\left|u_{2}(t)\right|+\left|u_{3}(t)\right| \\
& \leq 2 t^{-\sigma} \sup _{0.2 \leq s \leq 1}\|\phi(s)\|_{1}, \quad t \geq 1,
\end{aligned}
$$

where $u(t)=\left(u_{1}(t), u_{2}(t), u_{3}(t)\right)^{T}$ is the solution of $(67)$ initiated from $\phi \in \mathscr{C}\left([0.2,1], \mathbb{R}^{3}\right)$ and $\sigma$ is the unique positive solution of the equation $0.1273 \sigma-1+0.5613 e^{\sigma}=0$.

Remark 2. It needs to be pointed out that the impulsive instants are only selected as $2,4,6, \ldots$ in the simulation of this example to simplify the simulation, which is obviously not enough to illustrate the impulsive effect. In order to accurately characterize wider of impulses, the papers $[39,40]$ proposed the concepts of average dwell time and average impulsive interval. Moreover, the papers [41, 42] presented single impulsive controller and pinning impulsive stabilization criterion, respectively. Consequently, their methods are recommended to simulations with more general impulses. 


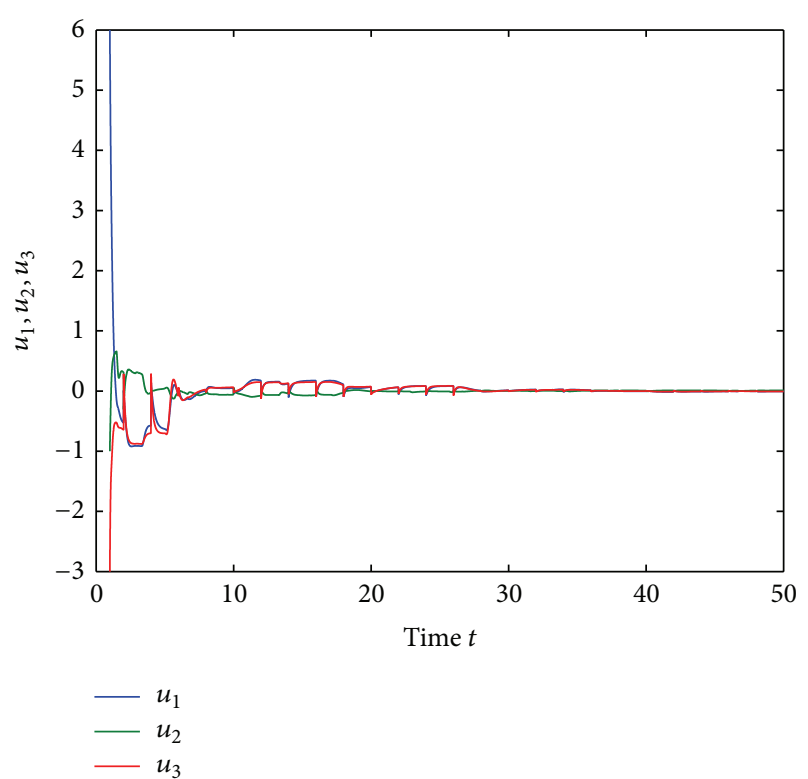

FIGURE 1: The simulation for the solution to the neural networks (67) at the impulsive moments $2,4,6, \ldots$.

\section{Conclusions and Further Work}

By means of relative nonlinear measure and transformation, this paper has discussed global asymptotic stability of impulsive cellular neural networks with proportional delays and partially Lipschitz activation functions. We have obtained the novel criterion of uniqueness and global asymptotic stability of the equilibrium point of this CNNs model. Our method does not require conventional assumptions on global Lipschitz continuity, boundedness, and monotonicity of activation functions and proportional delays to meet other requirements, which demonstrates that our criteria derived are less restrictive than some existing ones and that they are generalizations and improvements of some existing ones. Finally, the example with three cells has illustrated that our method is effective and that our results are correction.

Our method only requires the activation functions of the cellular networks to be partially Lipschitz continuous. The relative weak assumption makes our results applicable to more general engineering problems. In the future, we attempt to design a cellular neural networks model to solve optimization problems with some constraints, where unbounded (or nonmonotonic, nonglobally Lipspchitz continuous) activations are required such that these constraints are satisfied.

\section{Conflict of Interests}

The authors declare that there is no conflict of interests regarding the publication of this paper.

\section{Acknowledgments}

This work was supported by the Natural Science Foundation of China under the Contact (nos. 11201038, 11171043), Youth Science and Technology Nova Program of Shaanxi
Province under the Contact no. 2014KJXX-55, the Scientific Research Program Funded by Shaanxi Provincial Education Department under the Contact no. 2013JK0591, the Special Fund for Basic Scientific Research of Central Colleges in Changan University under the Contact (nos. 2013G2121017, CHD2012TD015), and Technology Foundation for Selected Overseas Chinese Scholar, Ministry of Personnel of China the Natural Science.

\section{References}

[1] L. O. Chua and L. Yang, "Cellular neural networks: theory," IEEE Transactions on Circuits and Systems, vol. 35, no. 10, pp. 1257$1272,1988$.

[2] L. O. Chua and L. Yang, "Cellular neural networks: applications," IEEE Transactions on Circuits and Systems, vol. 35, no. 10, pp. 1273-1290, 1988.

[3] P. P. Civalleri, M. Gilli, and L. Pandolfi, "On stability of cellular neural networks with delay," IEEE Transactions on Circuits and Systems. I. Fundamental Theory and Applications, vol. 40, no. 3, pp. 157-165, 1993.

[4] M. Gilli, M. Biey, and P. Checco, "Equilibrium analysis of cellular neural networks," IEEE Transactions on Circuits and Systems. I. Regular Papers, vol. 51, no. 5, pp. 903-912, 2004.

[5] W. Chen and W. X. Zheng, "A new method for complete stability analysis of cellular neural networks with time delay," IEEE Transactions on Neural Networks, vol. 21, no. 7, pp. 1126-1138, 2010.

[6] T. Kato and J. B. Mcleod, "The functional-differential equation," Bulletin of the American Mathematical Society, vol. 77, no. 2, pp. 891-937, 1971.

[7] L. Q. Zhou, "Delay-dependent exponential stability of Cellular neural networks with multiproportional delays," Neural Processing Letters, vol. 38, pp. 347-359, 2013.

[8] M. Tan, "Feedback stabilibzation of linear systems with proportional time delay," Information and Control, vol. 35, no. 6, pp. 690-694, 2006.

[9] A. Iserles and Y. Liu, "On neutral functional-differential equations with proportional delays," Journal of Mathematical Analysis and Applications, vol. 207, no. 1, pp. 73-95, 1997.

[10] Y. K. Liu, "Asymptotic behavior of functional differential equations with proportional time delays," European Journal of Applied Mathematics, vol. 35, no. 6, pp. 690-694, 1996.

[11] L. Li and L. Huang, "Equilibrium analysis for improved signal range model of delayed cellular neural networks," Neural Processing Letters, vol. 31, no. 3, pp. 1771-1794, 2010.

[12] P. Balasubramaniam, M. S. Ali, and S. Arik, "Global asymptotic stability of stochastic fuzzy cellular neural networks with multiple time-varying delays," Expert Systems with Applications, vol. 37, no. 12, pp. 7737-7744, 2010.

[13] P. Liu, "Delay-dependent global exponential robust stability for delayed cellular neural networks with time-varying delay," ISA Transactions, vol. 52, no. 6, pp. 711-716, 2013.

[14] Y. T. Zhang and Q. Luo, "Global exponential stability of impulsive cellular neural networks with time-varying delays via fixed point theory," http://www.advancesindifferenceequations .com/content/2013/1/23.

[15] B. Wu, Y. Liu, and J. Lu, "New results on global exponential stability for impulsive cellular neural networks with any bounded time-varying delays," Mathematical and Computer Modelling, vol. 55, no. 3-4, pp. 837-843, 2012. 
[16] I. M. Stamova and R. Ilarionov, "On global exponential stability for impulsive cellular neural networks with time-varying delays," Computers \& Mathematics with Applications, vol. 59, no. 11, pp. 3508-3515, 2010.

[17] Q. T. Gan, R. Xu, and P. H. Yang, "Stability analysis of stochastic fuzzy cellular neural networks with time-varying delays and reaction-diffusion terms," Neural Processing Letters, vol. 32, no. 1, pp. 45-57, 2010.

[18] S. Long and D. Xu, "Stability analysis of stochastic fuzzy cellular neural networks with time-varying delays," Neurocomputing, vol. 74, no. 14-15, pp. 2385-2391, 2011.

[19] Z. Feng and J. Lam, "Stability and dissipativity analysis of distributed delay cellular neural networks," IEEE Transactions on Neural Networks, vol. 22, no. 6, pp. 976-981, 2011.

[20] X. Song, X. Xin, and W. Huang, "Exponential stability of delayed and impulsive cellular neural networks with partially Lipschitz continuous activation functions," Neural Networks, vol. 29-30, pp. 80-90, 2012.

[21] C. Huang and J. Cao, "Almost sure exponential stability of stochastic cellular neural networks with unbounded distributed delays," Neurocomputing, vol. 72, no. 13-15, pp. 3352-3356, 2009.

[22] P. Balasubramaniam, M. Kalpana, and R. Rakkiyappan, "Existence and global asymptotic stability of fuzzy cellular neural networks with time delay in the leakage term and unbounded distributed delays," Circuits, Systems, and Signal Processing, vol. 30, no. 6, pp. 1595-1616, 2011.

[23] C. D. Zheng, Y. Wang, and Z. S. Wang, "Global stability of fuzzy cellular neural networks with mixed delays and leakage delay under impulsive perturbations," Circuits Systems Signal Process, vol. 33, no. 4, pp. 1067-1094, 2014.

[24] S. Ahmad and I. M. Stamova, "Global exponential stability for impulsive cellular neural networks with time-varying delays," Nonlinear Analysis: Theory, Methods \& Applications, vol. 69, no. 3, pp. 786-795, 2008.

[25] Y. T. Li and C. B. Yang, "Global exponential stability analysis on impulsive BAM neural networks with distributed delays," Journal of Mathematical Analysis and Applications, vol. 324, no. 2, pp. 1125-1139, 2006.

[26] B. Liu and L. Huang, "Global exponential stability of BAM neural networks with recent-history distributed delays and impulses," Neurocomputing, vol. 69, no. 16-18, pp. 2090-2096, 2006.

[27] S. Mohamad, K. Gopalsamy, and H. Akca, "Exponential stability of artificial neural networks with distributed delays and large impulses," Nonlinear Analysis: Real World Applications, vol. 9, no. 3, pp. 872-888, 2008.

[28] Z. W. Ping and J. G. Lu, "Global exponential stability of impulsive Cohen-Grossberg neural networks with continuously distributed delays," Chaos, Solitons and Fractals, vol. 41, no. 1, pp. 164-174, 2009.

[29] Y. Wang, W. Xiong, Q. Zhou, B. Xiao, and Y. Yu, "Global exponential stability of cellular neural networks with continuously distributed delays and impulses," Physics Letters A, vol. 350, no. 1-2, pp. 89-95, 2006.

[30] Q. H. Zhou, "Global exponential stability of BAM neural networks with distributed delays and impulses," Nonlinear Analysis: Real World Applications, vol. 10, no. 1, pp. 144-153, 2009.

[31] Z. T. Huang, X. S. Luo, and Q. G. Yang, "Global asymptotic stability analysis of bidirectional associative memory neural networks with distributed delays and impulse," Chaos, Solitons and Fractals, vol. 34, no. 3, pp. 878-885, 2007.
[32] E. Kaslik and S. Sivasundaram, "Multistability in impulsive hybrid Hopfield neural networks with distributed delays," Nonlinear Analysis: Real World Applications, vol. 12, no. 3, pp. 16401649, 2011.

[33] M. Forti and A. Tesi, "New conditions for global stability of neural networks with application to linear and quadratic programming problems," IEEE Transactions on Circuits and Systems I: Fundamental Theory and Applications, vol. 42, no. 7, pp. 354366, 1995.

[34] H. Qiao, J. G. Peng, and Z. B. Xu, "Nonlinear measures: a new approach to exponential stability analysis for Hopfield-type neural networks," IEEE Transactions on Neural Networks, vol. 12, no. 2, pp. 360-370, 2001.

[35] J. Peng, H. Qiao, and Z. Xu, "A new approach to stability of neural networks with time-varying delays," Neural Networks, vol. 15, no. 1, pp. 95-103, 2002.

[36] K. Gopalsamy, Stability and Oscillations in Delay Differential Equations of Populations Dynamics, vol. 74, Kluwer, Dodrecht, The Netherlands, 1992.

[37] B. Liu, "New convergence behavior of solutions to shunting inhibitory cellular neural networks with unbounded delays and time-varying coefficients," Applied Mathematical Modelling, vol. 33, no. 1, pp. 54-60, 2009.

[38] L. L. Wang and T. P. Chen, "Complete stability of cellular neural networks with unbounded time-varying delays," Neural Networks, vol. 36, pp. 11-17, 2012.

[39] J. Lu, D. W. C. Ho, and J. Cao, "A unified synchronization criterion for impulsive dynamical networks," Automatica, vol. 46, no. 7, pp. 1215-1221, 2010.

[40] J. Lu, J. Kurths, J. Cao, N. Mahdavi, and C. Huang, "Synchronization control for nonlinear stochastic dynamical networks: Pinning impulsive strategy," IEEE Transactions on Neural Networks and Learning Systems, vol. 23, no. 2, pp. 285-292, 2012.

[41] J. Lu, D. W. C. Ho, J. Cao, and J. Kurths, "Single impulsive controller for globally exponential synchronization of dynamical networks," Nonlinear Analysis. Real World Applications, vol. 14, no. 1, pp. 581-593, 2013.

[42] J. Q. Lu, Z. D. Wang, J. D. Cao, D. W. C. Ho, and J. Kurths, "Pinning impulsive stabilization of nonlinear dynamical networks with time-varying delay," International Journal of Bifurcation and Chaos, vol. 22, no. 7, Article ID 1250176, 12 pages, 2012. 


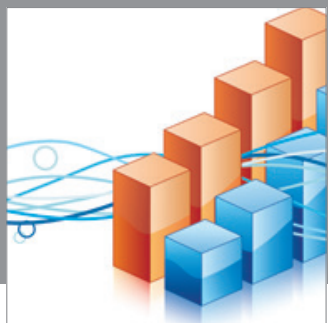

Advances in

Operations Research

mansans

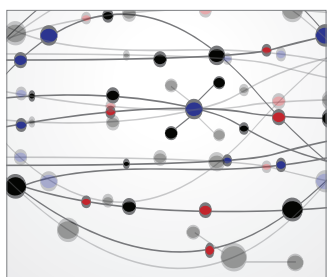

The Scientific World Journal
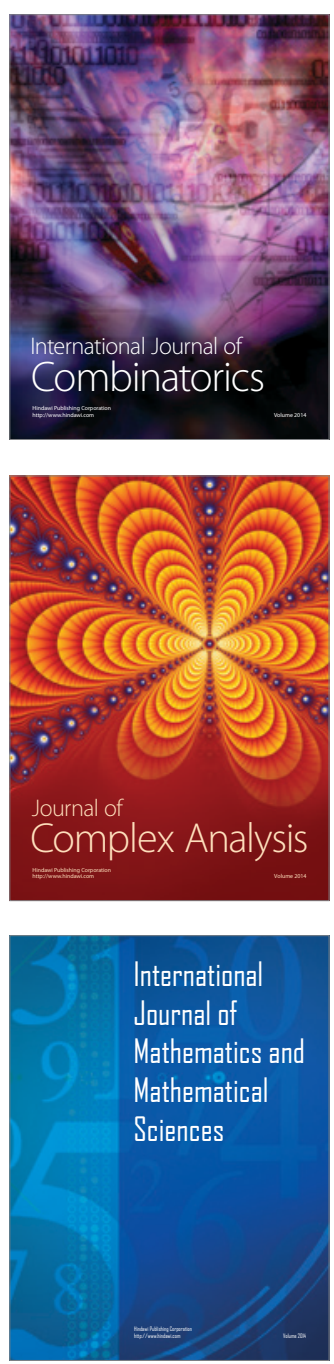
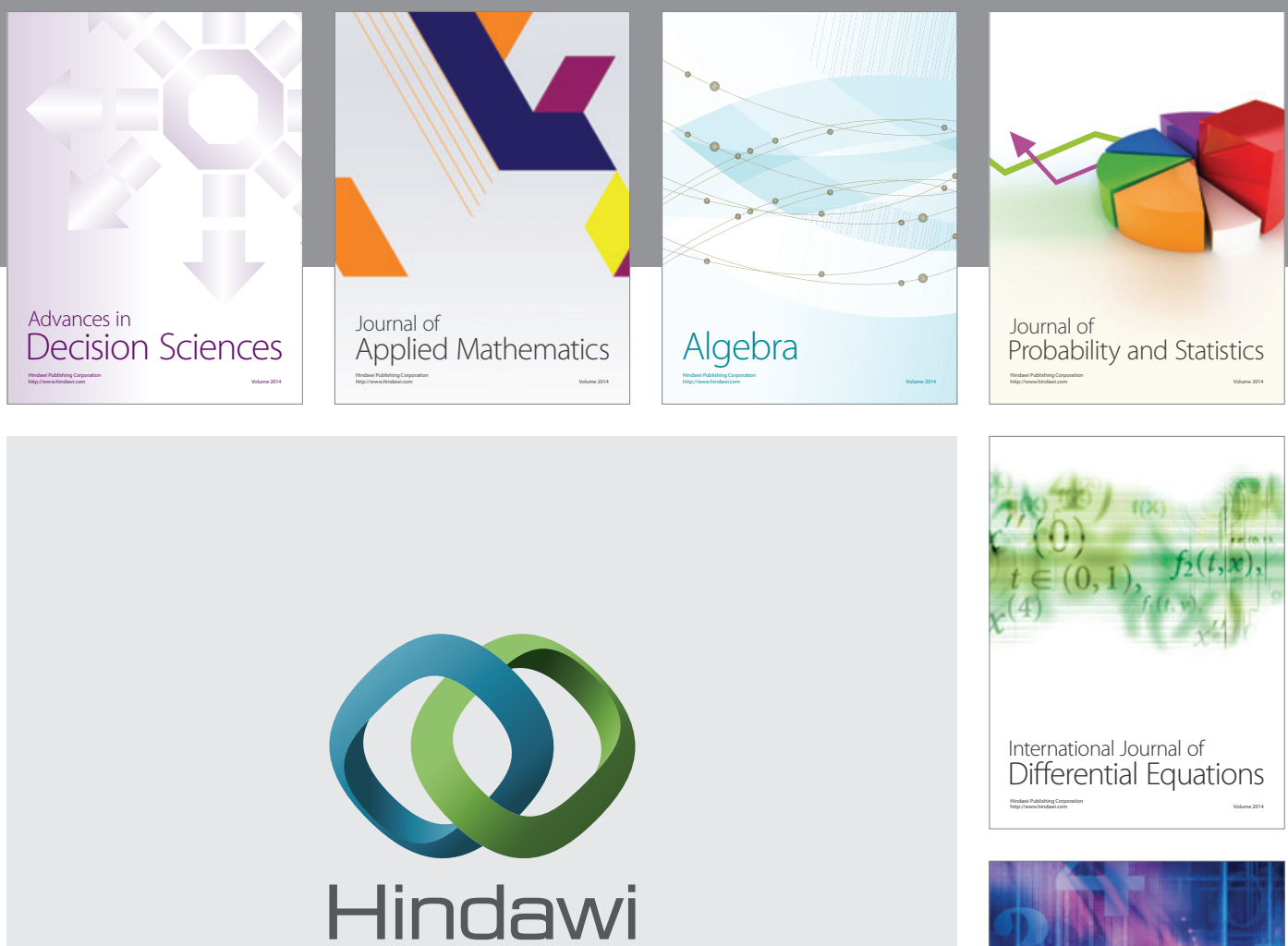

Submit your manuscripts at http://www.hindawi.com
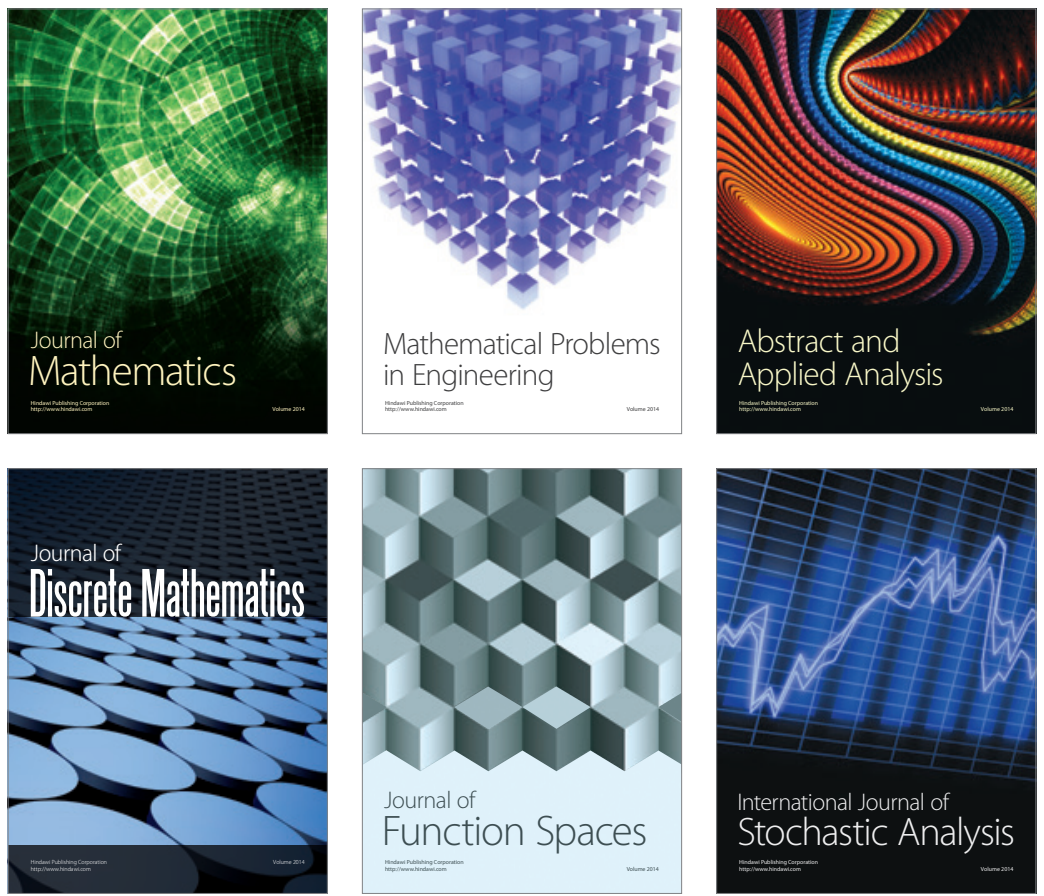

Journal of

Function Spaces

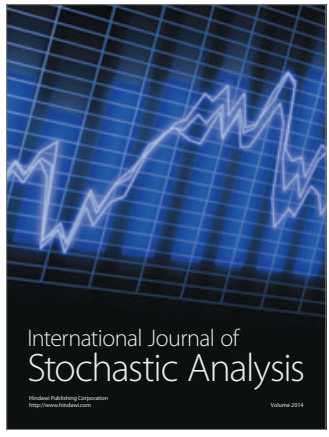

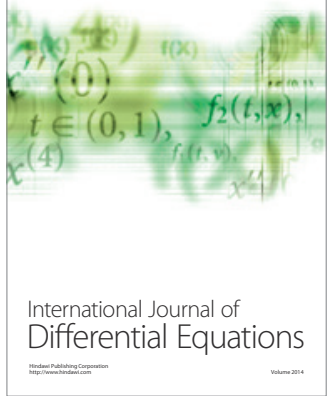
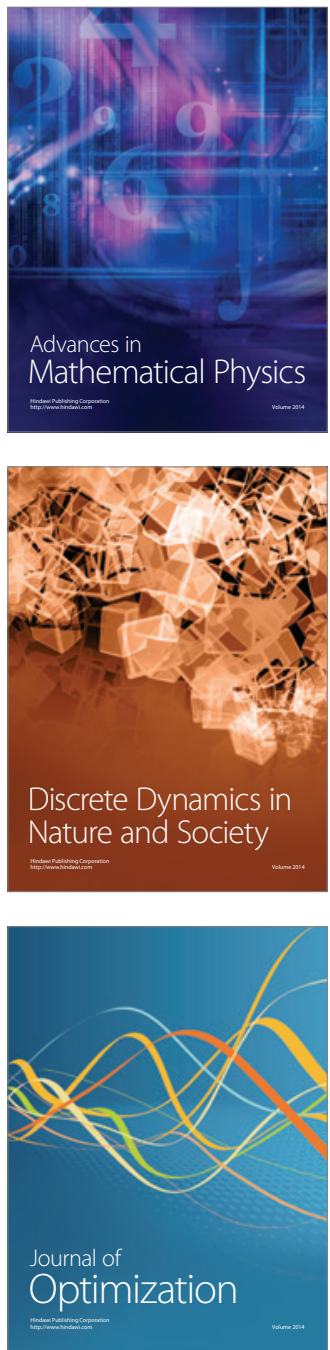